\title{
EFICÁCIA DA MOXIDECTINA, IVERMECTINA E ALBENDAZOLE CONTRA HELMINTOS GASTRINTESTINAIS EM PROPRIEDADES DE CRIAÇÃO CAPRINA E OVINA NO ESTADO DE PERNAMBUCO
}

\author{
Marilene Maria de Lima, ${ }^{1}$ Márcia Paula Oliveira Farias, ${ }^{2}$ Edenilze Teles Romeiro, ${ }^{3}$ Débora Rochelly \\ Alves Ferreira, ${ }^{4}$ Leucio Câmara Alves ${ }^{5}$ e Maria Aparecida da Gloria Faustino ${ }^{6}$
}

\author{
1. Professora adjunto da UFRPE-UAST, Recife-PE, BR. E-mail: lenelima@yahoo.com.br \\ 2. Doutoranda da UFRPE \\ 3. Pofessora adjunto da UFRPE \\ 4. Doutoranda da UFRPE \\ 5. Professor adjunto da UFRPE \\ 6. Professora adjunto da UFRPE.
}

RESUMO

Avaliou-se a eficácia de produtos anti-helmínticos à base de moxidectina, ivermectina e albendazole em propriedades de criação caprina e ovina, localizadas em municípios pertencentes ao estado de Pernambuco. Os animais eram de ambos os sexos, idades e raças variadas, criados em sistema semi-intensivo e intensivo. A eficácia dos produtos foi avaliada pelo teste de redução do número de ovos por grama de fezes e calculada pela fórmula: \%eficácia $=1-[(\mathrm{OPG}$ médio pós-tratamento/OPG médio antes do tratamento)] x 100, indicando ineficácia com índice inferior a $95 \%$. A moxidectina mostrou-se eficiente tanto em propriedade de criação caprina como ovina, a ivermectina apresentou melhor desempenho nos ovinos, enquanto o albendazole não foi eficaz para nenhuma das duas espécies. O gênero Haemonchus foi o mais prevalente nas coproculturas.

PALAVRAS-CHAVES: Anti-helmíntico, caprino, ovino, Pernambuco, resistência.

ABSTRACT EFFICACY OF MOXIDECTIN, IVERMECTIN AND ALBENDAZOL AGAINST GASTROINTESTINAL HELMINTHS IN
GOAT AND SHEEP FARMS FROM PERNAMBUCO STATE, BRAZIL

Anthelmintic products efficacy was evaluated within goat and sheep herds in municipalities belonging to the state of Pernambuco, Brazil. The animals were male and female of various ages and breeds, reared under semi-intensive and intensive system. The effectiveness of Moxidectin, ivermectin and albendazole drugs was detected using the fecal egg count reduction test (FECRT), calcu- lated by the formula: \%efficacy $=1$ - [(Mean FEC post-treatment/ Mean FEC pre-treatment)] x 100, indicating inefficiency with index lower than $95 \%$. Moxidectin was efficient in sheep and goat herds, ivermectin showed better performance in sheep, while albendazole was not effective for either species. Haemonchus sp. was the most prevalent in larval culture.

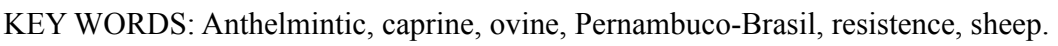

\section{INTRODUÇÃO}

A caprinovinocultura é uma atividade pecuária em expansão em todo Brasil. A produção desses ruminantes é prejudicada pelas helmintoses, que apre- sentam grande destaque, por causarem retardamento no desenvolvimento dos animais, levando à baixa produtividade e, consequentemente, elevadas perdas econômicas e até morte (SILVA et al., 2003). O controle do parasitismo por nematoides gastrintestinais é 
feito basicamente com a utilização de anti-helmínticos (MELO et al., 2004), gerando despesas com aquisição de drogas e o aumento de mão de obra (VIEIRA, 2003). Não obstante, devido aos custos elevados destes produtos, associado à falta de conhecimento básico no que tange à biologia e à epidemiologia dos parasitos, a maioria dos criadores não dosifica adequadamente seus rebanhos (VIEIRA \& XIMENES, 2001), favorecendo, assim, o surgimento da resistência pelos parasitos aos anti-helmínticos

A resistência a drogas anti-helmínticas é um fato crescente e difundido em todo mundo (CUNHA FILHO \& YAMAMURA, 1999). A indústria farmacêutica vem, historicamente, encarando este desafio com o desenvolvimento de drogas que incluem os modernos antiparasitários de amplo espectro e alto poder residual. Segundo VIEIRA \& CAVALCANTE (1998), a resistência anti-helmíntica é definida como o aumento significativo na habilidade de uma estirpe de parasitos para tolerar doses de uma droga que são letais para a maioria dos indivíduos de uma população da mesma espécie.

No Brasil, a resistência já foi relatada em várias regiões. No Ceará, VIEIRA \& CAVALCANTE (1999) observaram resistência aos anti-helmínticos do grupo dos imidazóis e benzimidazóis. Em Pernambuco, a resistência foi relatada por CHARLES et al. (1989) ao levamisole, albendazole e parbendazole. BARRETO \& SILVA (1999), em trabalhos realizados na Bahia, observaram também resistência aos anti-helmínticos albendazole e ivermectina. MELO et al. (2003), no estado do Ceará, observaram a ocorrência de resistência aos anti-helmínticos, oxfendazole, levamisole, ivermectina em nematoides de ovinos e caprinos.

As lactonas macrocíclicas têm sido amplamente usadas e sua eficácia relatada em várias regiões (PANKAVICH et al., 1992; TAYLOR et al., 1993; URIARTE et al., 1994; CUNHA FILHO \& YAMAMURA, 1999; LEONARDO et al., 2006), a exemplo da moxidectina que, dentre outras, segundo OLIVEIRA et al. (2002), apresenta a vantagem de persistência de eficácia.

Entre os parasitos mais resistentes destaca-se Haemonchus contortus. O primeiro caso de resistência desse parasito aos benzimidazóis ocorreu no Rio Grande do Sul (SANTOS \& GONÇALVES, 1967).

Diversos fatores contribuem para o aparecimento da resistência, destacando-se, dentre outros, o uso contínuo de anti-helmínticos pertencentes à mesma classe de drogas (WALLER, 1992) e o uso de subdose (VIEIRA \& CAVALCANTE, 1999), associado à falta de exames coproparasitológicos para monitoramento da eficácia dos produtos utilizados.

Os resultados alcançados com o uso dos produtos é a questão que leva os proprietários a duvidarem da eficácia. Portanto, o uso do teste de redução do número de ovos por grama de fezes (OPG) é uma ferramenta útil, principalmente nos casos em que os testes in vivo são economicamente inviáveis para os produtores. De acordo com ALBERTI et al. (2001), esse teste não necessita de uma equipe altamente treinada, recursos caros, ou de equipamentos sofisticados e, segundo VIEIRA \& CAVALCANTE (1998), é um método prático, rápido, não requer, necessariamente, o sacrifício dos animais e, por isso, deve ser implementado na propriedade, para monitorar a eficácia dos anti-helmínticos utilizados.

Pelo fato de a resistência anti-helmíntica constituir-se num dos principais fatores limitantes para a produção animal, uma vez que inviabiliza o controle efetivo da helmintose dos pequenos ruminantes, com reflexos negativos nos índices produtivos (VIEIRA, 2003), desenvolveu-se este trabalho para avaliar a eficácia de produtos à base de moxidectina, ivermectina e albendazole em propriedades de criação caprina e ovina.

\section{MATERIAL E MÉTODOS}

O estudo foi realizado em propriedades de criação caprina e ovina localizadas em municípios pertencentes ao estado de Pernambuco. Utilizaram-se caprinos e ovinos de ambos os sexos, idades e raças variadas, criados em sistema semi-intensivo e intensivo. Selecionaram-se propriedades cujos proprietários se propunham a manter o esquema necessário para a execução dos testes de eficácia. Os anti-helmínticos testados foram selecionados obedecendo-se às informações dos proprietários, as quais consistiam em falha dos produtos utilizados anteriormente, morte de animais, mesmo utilizando-se o tratamento anti-helmíntico e, em alguns casos, a escolha foi realizada com objetivo de monitoramento da eficácia do produto.

A via de aplicação bem como as dosagens dos anti-helmínticos seguiram a recomendação indicada 
pelo fabricante. Amostras de fezes foram coletadas sete dias antes do tratamento (dia -7), no dia do tratamento (dia 0 ), e sete dias após o tratamento (dia +7 ), sendo os resultados do $7^{\circ}$ dia utilizados para o cálculo de eficácia. Foi possível verificar a persistência de eficácia dos produtos em algumas propriedades estudadas. $\mathrm{O}$ número de animais utilizados variou de acordo com o efetivo de cada propriedade. Processaram-se as amostras para determinar o número de ovos por grama de fezes (OPG), segundo GORDON \& WHITLOCK (1939). Interpretou-se o grau de infecção para ovos tipo Strongyloidea segundo UENO \& GONÇALVES (1998). Para obtenção das larvas de terceiro estágio de nematoides, foi empregada a técnica de ROBERTS \& O'SULLIVAN (1950). Procedeu-se aos exames no Laboratório de Doenças Parasitárias dos Animais Domésticos, Departamento de Medicina Veterinária, Universidade Federal Rural de Pernambuco.

Para a análise estatística, calculou-se a média aritmética do OPG antes e depois do tratamento para cada grupo. A eficácia dos produtos foi avaliada pelo teste de redução do número de ovos por grama de fezes (EDWARDS et al., 1986), calculada pela fórmula: \% eficácia $=1$ - [(OPG médio pós-tratamento/OPG médio pré - tratamento)] x 100, indicando eficácia com índice maior ou igual a 95\% (COLES, 1992).

\section{RESULTADOS E DISCUSSÃO}

Em todas as propriedades referentes às duas espécies animais observou-se positividade para ovos tipo Strongyloidea nos exames anteriores aos tratamentos, com grau de infecção variando de leve a pesado tanto em caprinos quanto em ovinos (Tabela 2), corroborando os dados registrados por LIMA et al. (2003) e LIMA (2001) na Região Metropolitana de Recife. Trabalhos realizados por NEVES et al. (2005), com ovinos provenientes da Paraíba, e por SANTOS et al. (2004), com caprinos no Estado do Maranhão, relataram o mesmo grau de infecção ao OPG encontrado no presente estudo.

Os produtos à base de moxidectina apresentaram percentuais indicativos de eficácia tanto nos rebanhos caprinos quanto nos ovinos onde foram testados, semelhantes aos dados encontrados no Paraná por CUNHA FILHO \& YAMAMURA (1999) e LEONARDO et al. (2006), que observaram eficácia de $100 \%$ para moxidectina em caprinos e ovinos, respectivamente. No entanto, CUNHA FILHO et al. (1999), na Região de Londrina, PR, observaram eficácia de apenas $20 \%$ para moxidectina em ovinos e MILCZEWSKI et al. (2006) registraram resistência à moxidectina em 93,7\% das propriedades estudadas no estado do Paraná na espécie ovina.

Apesar de HOOKE et al. (1997) afirmarem que as vias de administração dos medicamentos interferem no desempenho da droga, no presente estudo, tanto a moxidectina aplicada pela via oral quanto a formulação injetável apresentaram eficácia semelhante.

Em relação aos rebanhos tratados com albendazole (Tabela 1), os percentuais de redução do OPG (30\% e 70,50\%) indicaram resistência. BARRETO \& SILVA (1999) registraram dados semelhantes no estado da Bahia, observando uma eficácia de 79,31\% em caprinos. A resistência ao albendazole também foi registrada em uma propriedade ovina, a qual apresentou eficácia de 75,72\% (Tabela 1). BARBOSA et al. (2004), utilizando o sulfóxido de albendazole em caprinos, verificaram um índice de $0 \%$. O albendazole, durante anos, foi um dos princípios ativos mais utilizados pelos proprietários, no Nordeste, contribuindo, assim, para o desenvolvimento da resistência.

A ivermectina apresentou eficácia de 67,33\% em caprinos, indicando resistência. BARRETO \& SILVA (1999) encontraram resultados semelhantes, ao observarem uma redução de OPG de $84,84 \%$ em caprinos no estado da Bahia. Em relação aos ovinos, registrou-se índice de 100\% (Tabela 1), corroborando os dados de MELO et al. (2003), que relataram redução de $100 \%$ na contagem de ovos nas fezes, após o tratamento com ivermectina em ovinos no estado do Ceará. Trata-se de índice que difere, no entanto, do constatado por BARRETO et al. (2004), em trabalho realizado em nove rebanhos ovinos no estado da Bahia, relatando ineficácia.

Os resultados obtidos nas propriedades em que foi possível verificar a persistência da eficácia dos produtos registram-se na Tabela 2. Em caprinos, a moxidectina manteve, quatorze dias após o tratamento, em Camocim de São Félix, percentual de 91,00\%, que, embora superior aos verificados para as demais propriedades, não atingiu o nível necessário indicativo de eficácia. Em ovinos, verificou-se redução de 99,70\%, em Recife, até 21 dias, concordando com TAYLOR et 
al. (1993), que observaram eficácia prolongada da moxidectina $1 \%$ em ovinos também por 21 dias. A ivermectina em ovinos apresentou persistência da eficácia até 21 dias em Vitória de Santo Antão (100\%). Este resultado relacionado à ivermectina em ovinos difere das observações de CUNHA FILHO \& YAMAMURA (1999), que obtiveram, aos 14 dias pós-tratamento, eficácia de $68,26 \%$, concluindo por resistência antihelmíntica a esta base.

TABELA 1. Resultado do teste de redução de OPG antes e depois (Dia +7) da aplicação dos anti-helmínticos em caprinos e ovinos criados em municípios localizados no estado de Pernambuco

\begin{tabular}{|c|c|c|c|c|c|}
\hline Produtos utilizados & Município & Espécie & $\begin{array}{c}\text { OPG médio } \\
\text { pré-tratamento }\end{array}$ & $\begin{array}{c}\text { OPG médio } \\
\text { pós-tratamento }\end{array}$ & Eficácia \% \\
\hline Moxidectina $1 \%$ & Sertânia & Caprina & 793,02 & 48,27 & 93,84 \\
\hline Moxidectina $1 \%$ & Paudalho & Caprina & 689,47 & 15,78 & 97,69 \\
\hline Moxidectina 1\% & $\begin{array}{c}\text { Camocim de São } \\
\text { Félix }\end{array}$ & Caprina & 661,5 & 69,2 & 90,0 \\
\hline Albendazole & Sertânia & Caprina & 1012,24 & 709,52 & 30 \\
\hline Albendazole & $\begin{array}{l}\text { Taquaritinga do } \\
\text { Norte }\end{array}$ & Caprina & 638,91 & 188,23 & 70,50 \\
\hline Ivermectina & Recife $^{a}$ & Caprina & 1.836 & 600 & 67,33 \\
\hline Moxidectina $1 \%$ & Recife $^{\mathrm{b}}$ & Ovina & 3445 & 9,09 & 99,8 \\
\hline Ivermectina $1 \%$ & $\begin{array}{l}\text { Vitória de Santo } \\
\text { Antão }\end{array}$ & Ovina & 4609,09 & 0 & 100 \\
\hline Albendazole & Garanhuns & Ovina & 1016,6666 & 255,5555 & 75,72 \\
\hline
\end{tabular}

TABELA 2. Resultados do teste de redução da contagem de ovos por grama de fezes em rebanhos caprinos e ovinos 14 e 21 dias após o tratamento

\begin{tabular}{|c|c|c|c|c|c|c|}
\hline Produtos & Municípios & Espécie & $\mathrm{OPG}+14$ & Eficácia \% & $\mathrm{OPG}+21$ & Eficácia \% \\
\hline Moxidectina $1 \%$ & Sertânia & Caprina & 92,59 & 88,18 & - & - \\
\hline Moxidectina $1 \%$ & Paudalho & Caprina & - & - & - & - \\
\hline Moxidectina $1 \%$ & Camocim de São Félix & Caprina & 61,5 & 91,00 & - & - \\
\hline Albendazole & Sertânia & Caprina & 793,02 & 21,66 & - & - \\
\hline Albendazole & Taquaritinga do Norte & Caprina & - & - & 840,90 & 31,16 \\
\hline Ivermectina & Recife & Caprina & 1500 & 48,10 & 1450 & 46,85 \\
\hline Moxidectina $1 \%$ & Recife & Ovina & 0,08 & 99,99 & 10,08 & 99,70 \\
\hline Ivermectina $1 \%$ & Vitória de Santo Antão & Ovina & 9,09 & 97,83 & 0 & 100 \\
\hline Albendazole & Garanhuns & Ovina & 263,16 & 74,12 & 93,33 & 73,12 \\
\hline
\end{tabular}

(-) Teste não realizado.

Os resultados das coproculturas qualitativas antes e depois dos tratamentos revelaram o Haemonchus contortus como o mais prevalente (Tabela 3), concordando com os achados de MELO (2004) e BARBOSA et al. (2004), no Ceará e em Uberlândia, respectivamente. Apesar da eficácia evidenciada, observou-se desenvolvimento de larvas de $H$. contortus em todas as propriedades para todos os anti-helmínticos testados. Para Trichostrongylus spp. e Oesophagostomum sp., coproculturas resultaram negativas em algumas proprie- dades de criação caprina. Dentre os ovinos, verificou-se positividade para as larvas do gênero Haemonchus, Trichostrongylus e Oesophagostomum em todas as propriedades e bases farmacológicas utilizadas. Os resultados obtidos em relação à moxidectina diferem dos achados de VERÍSSIMO et al. (2000), que obtiveram percentual de eficácia de $0 \%$ em rebanho ovino de Nova Odessa, SP, relatando o primeiro caso de Haemonchus spp. resistente à moxidectina diagnosticado pelo mesmo método aplicado no presente estudo. 
Nos rebanhos caprinos, os anti-helmínticos utilizados anteriormente pertenciam ao grupo das avermectinas, benzimidazóis e milbemicuna, enquanto nas propriedades de criação ovina foram ivermectina e albendazole. $\mathrm{O}$ uso de produtos à base de albendazole foi observado na maioria das propriedades analisadas, indicando ser a base anti-helmíntica mais conhecida pelos proprietários e, provavelmente, justifica os reduzidos valores obtidos para a eficácia deste composto no presente estudo. Segundo CUNHA FILHO \& YAMAMURA (1999), certas condições tornaram os benzimidazóis (especificamente o albendazole) o grupo químico mais utilizado pelos produtores, tais como a facilidade de administração, o relativo baixo custo, a grande variedade de marcas presentes no mercado, propiciando, assim, o aparecimento da resistência. A diversidade de produtos de mesma base no mercado, conforme WALLER (1992), induz os produtores a alternarem medicamentos, efetuando, porém, uma rotação de anti-helmínticos com o mesmo princípio ativo sem mudar, efetivamente, as diferentes classes de anti-helmíntico. Tais fatores podem ter sido os responsáveis pela situação existente nas propriedades ora em estudo.

O percentual aceitável de eficácia obtido para a ivermectina pode ser explicado pelo fato de seu uso, em comparação com os benzimidazóis, ter sido introduzido mais recentemente na propriedade estudada.

TABELA 3. Larvas de helmintos encontrados nas coproculturas sete dias antes e sete dias depois dos tratamentos realizados em caprinos e ovinos criados no estado de Pernambuco

\begin{tabular}{|c|c|c|c|c|c|c|c|}
\hline \multirow{3}{*}{ Produtos } & \multirow{3}{*}{ Espécie } & \multicolumn{6}{|c|}{ Coproculturas } \\
\hline & & \multicolumn{3}{|c|}{ Antes do tratamento } & \multicolumn{3}{|c|}{ Depois do tratamento } \\
\hline & & HAEM & TRIC & OESO & HAEM & TRIC & OESO \\
\hline Moxidectina $1 \%$ & Caprina & + & + & + & + & - & + \\
\hline Moxidectina 1\% & Caprina & + & + & - & + & + & - \\
\hline Moxidectina $1 \%$ & Caprina & + & + & - & + & + & - \\
\hline Albendazole & Caprina & + & + & + & + & - & - \\
\hline Albendazole & Caprina & + & + & + & + & + & \\
\hline Ivermectina & Caprina & + & + & + & + & + & + \\
\hline Moxidectina 1\% & Ovina & + & + & + & + & + & + \\
\hline Ivermectina & Ovina & + & + & + & + & + & + \\
\hline Albendazole & Ovina & + & + & + & + & + & + \\
\hline
\end{tabular}

HAEM - Haemonchus sp. TRIC - Trichostrongylus sp. OESO -Oesophagostomum spp.

(+) Positivo (-) negativo (.) Não foi realizado o exame.

\section{CONCLUSÕES}

Nas propriedades estudadas, o tratamento antihelmíntico para caprinos e ovinos deve ser implementado com endectocidas, preferentemente, à base de moxidectina $1 \%$, mantendo-se um monitoramento nas criações onde os produtos ainda apresentam potencialidade para o controle das infecções helmínticas.

\section{REFERÊNCIAS}

BARBOSA, F. C.; LUIZ, L.I.; TEIXEIRA, T. C. S.; AMORIM, L. L. Eficácia do sulfóxido albendazole e closantel no controle da verminose em caprinos. Revista Brasileira de Parasitologia Veterinária, v. 13, p. 268, 2004. Suplemento.

BARRETO, M. A.; SILVA, J. S. Avaliação da resistência antihelmíntica de nematódeos gastrintestinais em rebanhos caprinos do Estado da Bahia: resultados preliminares. In: SEMINÁRIO BRASILEIRO DE PARASITOLOGIA VETERINÁRIA, 11.; SEMINÁRIO DE PARASITOLOGIA VETERINÁRIA DOS PAÍSES DO MERCOSUL, 2.; SIMPÓSIO DE CONTROLE INTEGRADO DE PARASITOS DE BOVINOS, 1., 1999, Salvador. Anais... Salvador: Colégio Brasileiro de Parasitologia Veterinária, 1999. p. 160.

BARRETO, M. A.; ALMEIDA, M. A. O.; SILVA, A.; BITENCUR, C. P. Avaliação da resistência anti-helmíntica de nematódeos gastrintestinais em ovinos deslanados do estado da Bahia. Revista 
Brasileira de Parasitologia Veterinária, v. 13, p. 265, 2004. Suplemento.

COLES, G. C.; BAUER, C.; BORGSTEEDE, F. H. M.; GEERTS, S.; KLEI, T. R.; TAYLOR, M. A.; WALLER, P. J. World Association for the Advancement of Veterinary Parasitology (WAAVP): methods for the detection of anthelmintic resistance in nematodes of veterinary importance. Veterinary Parasitology, v. 44, p. 3544. 1992.

CUNHA FILHO, L. F.; YAMAMURA, M. H. Resistência a antihelmínticos em ovinos da região de Tamarana, Paraná, Brasil. UNOPAR. Ciência Biológica da Saúde, Londrina, v. 1, n. 1, p. 31-39, 1999.

CUNHA FILHO, L. F. C.; YAMAMURA, M. H.; PEREIRA, A. B. L. Resistência anti-helmíntica em ovinos da Região de londrina. In: SEMINÁRIO BRASILEIRO DE PARASITOLOGIA VETERINÁRIA, 11., Salvador, 1999. Bahia. Anais... Salvador: CBPV, 1999. p. 153.

CHARLES, T. P.; POMPEU, J.; MIRANDA D. B. Efficacy of three broad-spectrum anthelmintics against gastrointestinal nematoda infections of goats. Veterinary Parasitology, n. 34, p. 71-75, 1989.

DOS SANTOS, V. T.; GONÇALVES, P. C. Variação de estirpes de Haemonchus resistentes ao thiabendazole no Rio Grande do Sul. Revista da Faculdade de Agronomia e Veterinária, v. 9, p. 201-209, 1967.

EDWARDS, J. R.; WROTH, R.; CHANEET, G. C. Survey of anthelmintic resistance in Western Australia sheep flocks, prevalence. Australian Veterinary Journal, v. 63, n. 5, p. 135-138, 1986.

GORDON, H. Mcl.; WHITLOCK, H. V. A New technique for counting nematoda eggs in sheep faeces. Journal Commonwealth Science and Industry Organization, v. 12, n. 1, p. 50-52, 1939.

HOOKE, F. G.; CLEMENT, P.; DELL' OSA, D.; PORTER, R. M.; MacCOLL, D.; REW, R.S. Therapeutic and protective efficacy of doramectin injectable against gastrointestinal nematodes in cattle in New Zealand: a comparison with moxidectin and ivermectin pour-on formulations. Veterinary Parasitology, n. 72, p. 43-51, 1997.

LANUSSE C. E.; PRICHARD R. K. Relationship pharmacological properties and clinical efficacy of ruminant anthelmtics. Veterinary Parasitology, v. 49, n. 2-4, p. 123-158, 1993.

LEONARDO, J. M. L. O.; AGULHON, A. Z.; PICOLI, F. M.; GRACIANO, T. Eficácia de anti-helmínticos em caprinos naturalmente parasitados. In: CONGRESSO BRASILEIRO DE PARASITOLOGIA VETERINÁRIA, 2., SIMPÓSIO LATINOAMERICANO DE RICKETTSIOSES, 14., 2006, Ribeirão Preto. Anais... Ribeirão Preto: [s.n.], 2006. p. 296.

MELO, A. C. F. L.; REIS, I. S.; BEVILAQUA, C. M. L.; VIEIRA, L. S.; ECHEVARRIA, F. A. M.; MELO, L. M. Nematódeos resistentes a anti-helmíntico em rebanhos de ovinos e caprinos do estado do Ceará, Brasil. Ciência Rural, v. 33, n. 2, p. 339-344, 2003.
MELO, A. C. F. L. Resistência a anti-helmíntico em nematóides gastrintestinais de ovinos e caprinos na região do Baixo e Médio Jaguaribe. Ciência Animal, v. 14, n. 1, p. 62, 2004.

MELO, A. C. F. L.; RONDOMN, S.; REIS, I. S.; F. C. M; BEVILAQUA, C. M. L. Desenvolvimento da resistência ao oxfendazol em propriedades rurais de ovinos na região do Baixo e Médio Jaguaribe, Ceará, Brasil. Revista Brasileira de Parasitologia Veterinária, v. 13, n. 4, p. 137-141, 2004.

MILCZEWSKI, V.; SOTOMAIOR, C.S.;MORAES, F.R.; SCHWARTZ, M. G.; CALDAS, J. S.; THOMAZSOCCOL, V. Resistência anti-helmíntica em rebanhos ovinos e caprinos do estado do Paraná. In: CONGRESSO BRASILEIRODE PARASITOLOGIA VETERINÁRIA, 2.; SIMPÓSIO LATINO-AMERICANO DE RICKETTSIOSES, 14., 2006, Ribeirão Preto. Anais... Ribeirão Preto: [s.n.], 2006. p. 289.

MOLENTO, M. B. Resistência de helmintos em ovinos e caprinos. Revista Brasileira de Parasitologia Veterinária, Rio de Janeiro, v. 13 , p. $82-87,2004$. Suplemento.

NEVES, M. L. M. W.; FAUSTINO, M. A. G.; ALVES, L. C.; TEIXEIRA, W. C.; LIMA, M. M.; OLIVEIRA, M. P. B.;VBATISTA, A. V. M.; MEDEIROS, E. S.; AGUIAR, S. R. Eficácia anti-helmíntica da ivermectina $1 \%$ em ovinos provenientes do município de Gurjão, PB. In: JORNADA DE ENSINO, PESQUISA E EXTENSÃO DA UNIVERSIDADE FEDERAL RURAL DE PERNAMBUCO, 5. 2005, Recife. Anais... Recife, 2005. 1 CD-ROM.

PANKAVICH, J. A.; BERGER H.; SIMKINS, K. Efficacy of moxidectin, nemadectin and ivermectin against an ivermectin-resistant strain of Haemonchus contortus in sheep. Veterinary Record, v. 130, p. 241-243, 1992.

ROBERTS, F. H. S.; O'SULLIVAN, J. P. Methods for egg counts and larval cultures for strongyles infesting the gastrointestinal tract of cattle. Australian Journal of Agricultural Research, v. 1, p. 99-102, 1950.

SANTOS, A. C. G.; WAQUIM, M. A. M.; PEREIRA, L. A.; GUERRA, R. M. S. N. C.; FEITOSA, M. L. T.; TEIXEIRA, W. C.; CHAVES, E. P. Avaliação preliminar do parasitismo por helmintos gastrintestinais em caprinos da Baixada Maranhense. Revista Brasileira de Medicina Veterinária, v. 13, p. 264, 2004.

SILVA, W. W.; BEVILAQUA, C. M. L.; RODRIGUES, M. L. Variação sazonal de nematóides gastrintestinais em caprinos traçadores no semi-árido paraibano, Brasil. Revista Brasileira de Parasitologia Veterinária, v. 12, n. 1, p. 71-75, 2003.

TAYLOR, S. M.; EDGAR, H.; KENNY, J. Prophylactic efficacy of moxidectin for periparturient ewes and mid-summer lambs. Veterinary Record, v. 133, n. 11, p. 270-271, 1993.

UENO, H.; GONÇALVES, P. C. Manual para diagnóstico das helmintoses de ruminantes, 4. ed. Tokyo: Japan International Cooperation Agency, 1998. 143 p.

URIARTE J.; GRACIA M. J.; ALMEIDA S. Efficacy of moxidectin against gastrointestinal nematode infections in sheep. Veterinary Parasitology, v. 51, p. 301-305, 1994. 
VERÍSSIMO, C. J.; OLIVEIRA. S. M.; ESPOSITO FILHA, E.; REBOUÇAS, M. M.; CUNHA, SANTOS, L. E.; SARTORI, M.; LARA, M. A. C. E. A. Eficácia de anti-helmíntico em uma ovinocultura no estado de São Paulo: relato de Haemonchus spp., resistentes à moxidectina. Arquivo do Instituto Biológico, São Paulo, v. 67, p. 1-145, 2000. Suplemento.

VIEIRA, L. S. Alternativas de controle da verminose gastrintestinal dos pequenos ruminantes. In: CONGRESSO PERNAMBUCANO DE MEDICINA VETERINÁRIA, 5., 2003, Recife. Anais... Recife: Sociedade Pernambucana de Medicina Veterinária. 2003. p. 236-246.

VIEIRA, L. S.; CAVALCANTE, A. C. R. Resistência anti-helmíntica em nematódeos gastrintestinais de caprinos. Revista Brasileira de Medicina Veterinária, v. 20, n. 3, p. 112-117, 1998.
VIEIRA, L. S.; CAVALCANTE, A. C. R. Resistência anti-helmíntica em rebanhos caprinos no Estado do Ceará. Pesquisa Veterinária Brasileira, Brasília, v. 19, n. 3/4, p. 99-103, 1999.

VIEIRA, L. S.; XIMENES, L. J. F. Resistência genética ao parasitismo por nematódeos gastrintestinais em pequenos ruminantes no Brasil: panorama atual. Sobral, Embrapa Caprinos. 2001. 20 p. (Documentos, 36).

WALLER, P. J. Resistência dos nematóides aos anti-helmínticos na Austrália. A Hora Veterinária, v. 12, n. 69, p. 24-26,1992.

Protocolado em: 29 mar. 2007. Aceito em: 21 set. 2009. 